Interdisziplinäre Arbeitsgruppe Gentechnologiebericht:

Sina Bartfeld, Stephan Clemens, Tobias J. Erb, Heiner Fangerau, Boris Fehse, Jürgen Hampel, Ferdinand Hucho, Martin Korte, Stefan Mundlos, Jens Reich, Silke Schicktanz, Jochen Taupitz, Jörn Walter, Eva C. Winkler und Martin Zenke

\title{
Kurzfassung der Handlungsempfehlungen der IAG Gentechnologiebericht ${ }^{1}$
}

\section{Monitoring der Gentechnologien}

Die gesellschaftliche Relevanz gentechnologischer Entwicklungen hat sich letzten Jahren in rasant fortschreitenden Technologieentwicklungen mit disruptivem Potenzial und weltweit geführten Debatten z. B. um Keimbahninterventionen und Embryoide widergespiegelt. Aus diesem Grund bleiben ein interdisziplinäres Monitoring der Gentechnologien und die Förderung gesellschaftlicher Diskurse hochaktuelle Aufgaben. Die IAG Gentechnologiebericht empfiehlt daher, gentechnologische Verfahren, Forschungen und Anwendungen sowie ihre ethischen und gesellschaftspolitischen Dimensionen auch weiterhin interdisziplinär und international zu reflektieren und wo nötig, konkrete Handlungsempfehlungen zu entwickeln. Im Folgenden sind zu den Themenfeldern Stammzellforschung und Organoidtechnologie, Gentherapie, Gendiagnostik, Epigenetik, Grüne Gentechnik, Einzelzellanalyse und synthetische Biologie Handlungsempfehlungen aus Sicht der Mitglieder der IAG Gentechnologiebericht wiedergegeben.

\section{Handlungsempfehlungen zur Stammzellforschung}

- Bedeutung der Stammzellforschung: Die Stammzellforschung hat durch die neuen Techniken, patienten- und krankheitsspezifische Stammzellen aus normalen Körperzellen künstlich herstellen zu können, einen enormen Entwicklungsschub bekommen. Damit ergeben sich neue Chancen für die regenerative und personalisierte Medizin, Gen- und Biotechnologie. Eine exzellente Grundlagenforschung und kliniknahe Forschung sind der Schlüssel für eine erfolgreiche Translation von stammzellbasierten

1 Dies ist eine Kurzfassung der aus der Sicht der Mitglieder der IAG wichtigsten Empfehlungen basierend auf den folgenden ausführlichen „Handlungsempfehlungen zu den Themenfeldern der IAG Gentechnologiebericht". 
Verfahren in Medizin, Gen- und Biotechnologie und sollten daher gezielt und langfristig gefördert werden.

- Genome-Editing von Stammzellen: Die neuen Techniken des Genome-Editing sollten konsequent und langfristig erforscht werden, da sie neue Möglichkeiten zur patientenspezifischen Therapie und Medikamentenentwicklung (personalisierte Medizin, Präzisionsmedizin) für bislang nicht oder nur schwer therapierbare Erkrankungen erwarten lassen. Gleichzeitig sollten Sicherheits- und Risikoaspekte möglicher Anwendungen des Genome-Editing gründlich erforscht werden, da nur so eine fachkompetente Beurteilung und Abwägung der Chancen und Risiken erfolgen kann.

- Therapeutische Anwendungen humaner pluripotenter Stammzellen: Es ist zu erwarten, dass die derzeit weltweit durchgeführten klinischen Studien mit aus humanen embryonalen Stammzellen (hES-Zellen) abgeleiteten Zellprodukten in absehbarer Zeit in erfolgreiche Therapien münden werden. Der Gesetzgeber in Deutschland sollte Patientinnen und Patienten im Inland - allein schon wegen ihres Grundrechts auf Leben und körperliche Unversehrtheit - diese Therapien nicht vorenthalten.

- Ungeprüfte Stammzelltherapien: Wir sehen mit Besorgnis die in zunehmendem Maße international angebotenen ungeprüften Behandlungsangebote mit Stammzellen, die Menschen in häufig verzweifelten Lebenssituationen ansprechen wollen. Wissenschaftlich fundierte Informationsangebote für Patientinnen und Patienten über klinisch geprüfte Behandlungsmöglichkeiten mit Stammzellen sollten daher vorgehalten und langfristig gefördert werden.

- Ethische und rechtliche Aspekte der Forschung an humanen embryonalen Stammzellen: Die IAG empfiehlt eine Aufhebung des durch das Stammzellgesetz festgelegten Stichtags oder zumindest die Einführung eines gleitenden Stichtags oder eine Einzelfallprüfung. Auch die Einfuhr und die Verwendung von hES-Zellen sollten nicht nur zu Forschungszwecken, sondern auch zu diagnostischen, präventiven und therapeutischen Zwecken zulässig sein.

- Patentierung von auf humanen embryonalen Stammzellen basierenden Verfahren: Die europäische Regelung zur Patentierung von hES-Zellen und von auf hES-Zellen basierenden Verfahren und Produkten ist problematisch. Sie führt zudem zu rechtlichen Inkonsistenzen mit den Regelungen in fast allen EU-Mitgliedsstaaten, die Forschung an hES-Zellen und in vielen Mitgliedstaaten auch die kommerzielle Verwendung hES-Zell-basierter Verfahren und Produkte erlauben.

\section{Handlungsempfehlungen zur Organoidtechnologie}

- Die Bedeutung der Organoidtechnologie für die Biomedizin: Durch die Möglichkeit der Nutzung von Organoiden als individuelle Krankheitsmodelle hat die Organoidtechno- 
logie ein hohes Potenzial für die personalisierte Medizin. Translationale Forschung und klinische Studien mit anderen organoidbasierten personalisierten Therapien, bspw. in der Krebstherapie, sollten gezielt gefördert werden. Auch pharmazeutisch orientierte Grundlagenforschung und Kooperationen zwischen Forschung und Pharmaindustrie sollten unterstützt werden.

- Ethische und rechtliche Aspekte der Forschung an Hirnorganoiden: Nach derzeitigem Wissensstand ist Hirnorganoiden ein Bewusstsein und entsprechend ein Schutzanspruch unbedingt abzusprechen; selbst rudimentäre Formen von Bewusstsein werden Hirnorganoide auch bei weiteren Fortschritten der Forschung auf absehbare Zeit nicht entwickeln können. Vor diesem Hintergrund stehen der grundrechtlich geschützten Wissenschaftsfreiheit abgesehen von Rechten der Spenderinnen und Spender der für die Herstellung der Hirnorganoide verwendeten Ausgangszellen und abgesehen von Belangen des Tierschutzes, die beide gleichermaßen vom geltenden Recht hinreichend gewahrt sind, keine Belange von verfassungsrechtlichem Gewicht gegenüber. Weitergehende Beschränkungen der Forschung an und mit Hirnorganoiden sind deshalb auf absehbare Zeit nicht zu rechtfertigen.

- Ethische und rechtliche Aspekte der Forschung an Embryoiden: Es müssen klare rechtliche Rahmenbedingungen für die Embryoidforschung geschaffen werden, die der Wissenschaftsfreiheit angemessen Rechnung tragen, und bestehende Gesetze zur Forschung an menschlichen Embryonen ihrerseits vor diesem Hintergrund überprüft werden. Dringend revisionsbedürftig ist z. B. das strikte Verbot der Forschung mit menschlichen Embryonen. Sie sollte in bestimmtem Umfang auch in Deutschland erlaubt werden, und zwar - nach erfolgter Zustimmung der biologischen Eltern zumindest mit Embryonen, die zwar für Fortpflanzungszwecke erzeugt wurden, aber endgültig nicht mehr dafür verwendet werden und deshalb andernfalls verworfen werden. Es sollte auch eine zukünftig unter Umständen möglich werdende Nutzung von Embryoiden im Reproduktionskontext mit Bedacht und in Anbetracht des Wohls des dabei entstehenden Menschen explizit verboten werden.

\section{Handlungsempfehlungen zur Epigenetik}

- Epigenetik und individuelle Anpassung: Die Erforschung der Ursachen und Folgen epigenetischer Veränderungen im Rahmen kontrollierter, populationsbasierter Vergleichsstudien ist von großer lebenswissenschaftlicher und gesellschaftlicher Bedeutung und ein wichtiger Baustein für die Gesundheitsvorsorge. Derartige Studien sollten durch eine interdisziplinäre psychosoziale und ethische Begleitung ergänzt werden, um so über die Bedeutung möglicher Gesundheitsrisiken und die Problematik damit verbundener individueller Verantwortungszuschreibung zu reflektieren. 
- Epigenetische Diagnostik, Therapie- und Interventionsansätze: Die Entwicklung geeigneter Tests, Wirkstoffe und Methoden für epigenetische Diagnoseverfahren sollte intensiv unterstützt werden. Gleiches gilt für die Entwicklung neuer epigenetischer Therapieansätze und -verfahren.

- Epigenomforschung: Es wird notwendig sein, diese neue Forschungsaktivität national und international nachhaltig zu erhalten und mit den neuen Entwicklungen der Einzelzellanalytik zusammenzuführen. Darüber hinaus werden auch die ethischen Fragen zur Epigenomforschung und zu neueren Verfahren wie dem EpigenomeEditing untersucht werden müssen.

- Epigenetik und Ethik: Aus ethischer, rechtlicher und soziologischer Sicht ist ein interdisziplinär stattfindender Diskurs um epigenetische Daten und Konzepte breit zu führen. Hierzu müssen geeignete Foren, Strukturen und Institutionen identifiziert werden, in denen eine zielgruppenbezogene, sachlich fundierte, kritische Auseinandersetzung über gesellschaftliche Auswirkungen der Forschungsergebnisse zur epigenetischen Vererbbarkeit wie der Möglichkeiten und Risiken epigenomischer Eingriffe geführt werden kann. Die wissenschaftlichen Akademien könnten hierzu einen wesentlichen Beitrag leisten.

\section{Handlungsempfehlungen zur Gendiagnostik}

- Technologieentwicklung und ihre Anwendung: Der technologische Fortschritt in der Genomanalyse hat zu einer massiven Ausweitung der Anwendungsmöglichkeiten geführt. Es ist davon auszugehen, dass diese Entwicklung noch lange kein Ende gefunden hat und die Kosten für Genomanalysen weiter sinken werden. Deutschland muss an dieser Technologieentwicklung aktiv beteiligt sein, sowohl was die Technologien selbst, als auch was die Anwendung in der Medizin betrifft. Für die Anwendungen der neuen Sequenziermethoden bedarf es eines flexiblen und innovativen Einsatzes von Forschungsgeldern und, in der klinischen Anwendung, von Erstattungsregelungen. Derzeit wird nicht das beste Verfahren erstattet, sondern das, welches durch den einheitlichen Bewertungsmaßstab (EBM) vorgegeben ist. Dies wirkt sich als Innovationsbremse für neue Verfahren aus. Für die Erprobung von neuen Verfahren der Genomanalyse in der Klinik ergibt sich zwischen Forschung und Erstattung durch die Kassen eine Finanzierungslücke, die für eine erfolgreiche Translation geschlossen werden muss.

- Datenverarbeitung und -kommunikation verbessern: Die Möglichkeit, ganze Genome in kurzer Zeit für einen geringen Preis analysieren zu können, wird viele Bereiche der Medizin beeinflussen. Genome sind riesige Datenspeicher und ihre Information muss auch entsprechend verarbeitet werden. Es ist daher unerlässlich, dass neben 
der neusten Technologie zur Datengenerierung auch eine entsprechend leistungsfähige Datenverarbeitung zur Verfügung steht. Datenbanken, die die genomischen Daten speichern und vergleichen, sind für eine erfolgreiche Anwendung daher unerlässlich.

- Angemessene Versorgung und Anwendung sichern: Um ein hohes Maß an Behandlungsqualität in der genomischen Diagnostik zu erreichen, bedarf es einer stringenten, strukturierten und abgestimmten Vorgehensweise unter klinischen Expertinnen und Experten mit Spezialwissen aus verschiedenen Disziplinen, idealerweise in enger Verknüpfung mit Forschung. So sollte ein strukturierter Patientenpfad die Ratsuchenden erst an ein Zentrum führen, wo anhand der vorliegenden medizinischen Befunde von Expertinnen und Experten über das geeignete diagnostische Vorgehen entschieden wird. Diese Herausforderung sollte entsprechend qualifizierten Zentren übertragen werden. Eine vollständige Kommerzialisierung birgt die Gefahr einer Indikationsinflation und der Produktion von unnötigen Daten, was hohe Kosten verursacht, aber nicht zum Patientenwohl beiträgt.

- Die medizinische Anwendung und Translation in die Klinik bedarf speziell ausgebildeter Ärztinnen und Ärzte sowie Wissenschaftlerinnen und Wissenschaftler: Die Interpretation genomischer Daten ist kompliziert. Die Interpretation von Varianten, ihre klinische Einordnung und ihre Bedeutung für das Individuum bedürfen einer besonderen Beratung, die Teil des diagnostischen Programms darstellen sollte. Dies beinhaltet die Information und Beratung zu Ergebnissen, die mit der ursprünglichen Fragestellung nicht in Zusammenhang stehen, die jedoch für die Gesundheit und die weitere Lebensführung eine entscheidende Rolle spielen können.

- Ethische Aspekte und das Gendiagnostikgesetz: Das Gendiagnostikgesetz (GenDG), das im April 2009 vom Bundestag verabschiedet wurde, hat zur Akzeptanz genetischer Untersuchungen in der Bevölkerung beigetragen. Durch den seit Inkrafttreten des Gesetzes stattgefundenen technologischen Fortschritt sind allerdings einige der Regelungen nicht mehr zeitgemäß. Die im Gesetz ursprünglich vorgesehene Untersuchung einzelner weniger Gene wird jetzt durch Exomsequenzierung oder Ganzgenomsequenzierung abgelöst. Hieraus ergeben sich neue Herausforderungen. Das Gendiagnostikgesetz muss entsprechend überarbeitet und aktualisiert werden und die Regelungen an die neuen diagnostischen Möglichkeiten angepasst werden. Wie mit genomischen Daten in Zukunft umgegangen wird, bedarf einer ethischen und gesellschaftlichen Diskussion. 


\section{Handlungsempfehlungen zur somatischen Gentherapie}

- Forschung in Deutschland: Angesichts der hohen Preise für Gentherapeutika sollten im Interesse der Patientinnen und Patienten klare Regelungen getroffen werden, um einen breiten Zugang zu neuartigen, evidenzbasierten Therapien, deren Entwicklung über viele Jahre mit Steuermitteln gefördert wurde, sicherzustellen. Mit dem Ziel der effizienteren klinischen Umsetzung der erfolgreichen akademischen Gentherapieforschung in Deutschland empfiehlt die IAG die Implementierung strukturierter Programme zur Förderung der Translation innovativer zell- und gentherapeutischer Ansätze. Zudem könnten bundesweite Kompetenzzentren analog zu den Deutschen Gesundheitszentren einen Beitrag zur koordinierten Entwicklung der Gentherapie leisten.

- Keimbahninterventionen: Die IAG hält Keimbahninterventionen durch Genome-Editing mit Auswirkungen auf geborene Menschen für derzeit nicht vertretbar und sieht auch, zumindest vor dem Hintergrund der deutschen Rechtslage zur PID, praktisch kaum eine medizinische Indikation für eine solche Anwendung. Das Verbot von Keimbahninterventionen in Deutschland ergibt sich aus dem Embryonenschutzgesetz. Allerdings ist nicht ausgeschlossen, dass das bestehende Verbot der Keimbahninterventionen auf Basis der Fortschritte z. B. im Bereich induzierter pluripotenter Stammzellen und deren Differenzierbarkeit in Keimbahnzellen, technisch umgangen werden kann. Dies müsste bei einer möglichen Novellierung des Embryonenschutzgesetzes beachtet werden.

\section{Handlungsempfehlungen zur Grünen Gentechnologie}

- Anwendung und Regulierung: In einem ersten Schritt sollten die Definitionen genetisch veränderter Organismen modifiziert werden und genomeditierte Pflanzen mit Veränderungen, die nicht von natürlich auftretenden zu unterscheiden sind, von der Regulierung nach Direktive 2001/18/EC ausgenommen werden. Vergleichbares ist in den meisten Industrieländern (z. B. USA, Kanada, Australien, Japan, Brasilien und Argentinien) absehbar oder bereits geschehen. In einem zweiten Schritt sollte ein völlig neues Regelwerk entwickelt werden, das stärker auf der Beurteilung der Eigenschaften des Produkts basiert statt auf der verwendeten Methode der Produktentwicklung; potenzielle Risiken sollten auf der Grundlage wissenschaftlicher Evidenz beurteilt werden.

- Innovation: Die Grundlagenforschung z. B. an pflanzlichen Resistenz- oder Stresstoleranzmechanismen sollte weiterhin öffentlich gefördert werden, um züchterische Innovationen für gesündere Lebensmittel und nachhaltigere Produktion voranzubringen. 
- Forschungsfreiheit: Eine sehr nachteilige Einschränkung der Grundlagenforschung in Deutschland ist die faktische Verhinderung von kontrollierten Feldversuchen. Ähnlich wie in der Schweiz sollte eine zentrale Einrichtung aufgebaut werden, um wissenschaftliche Feldstudien mit genetisch veränderten Pflanzen ohne Bedrohung durch Vandalismus durchführen zu können.

- Diversität: Vor allem in Ländern des Globalen Südens sollte der Aufbau von Kapazitäten unterstützt werden, die die Forschung an neuen Züchtungsmethoden für die Verbesserung von unter den örtlichen Bedingungen wichtigen Nutzpflanzen (,orphan crops") ermöglichen. So können auch Abhängigkeiten von global operierenden Unternehmen und Patenten verringert werden.

\section{Handlungsempfehlungen zur synthetischen Biologie}

- Die Rolle der synthetischen Biologie in der Bioökonomie: Um den Übergang von einer fossilbasierten Synthesechemie zu einer nachhaltigen Bioökonomie zu ermöglichen, wird es notwendig sein, noch mehr als bisher synthetisch-biologische Ansätze und Technologien gezielt zu fördern und frühzeitig finanzielle und steuerliche Anreize zu setzen. Im Rahmen europäischer Anstrengungen (European Green Deal) sollte Deutschland als bedeutender Pharma-, Chemie- und Biotechnologiestandort für die Entwicklung einer nachhaltigen, modernen Bioökonomie eine führende Rolle übernehmen.

- Standardisierung in der synthetischen Biologie: Zusätzlich zu entsprechenden Förderprogrammen, die dem Aufbau einer nationalen bzw. europäischen synthetischen Biologielandschaft dienen, sollten sichere Forschungs- bzw. Industriestandards etabliert werden, um einen Übergang von der traditionellen Bio(techno)logie in eine digitalisierte und automatisierte biotechnologische Forschungs- und Produktionslandschaft zu gestalten. Hier könnte unter Berücksichtigung langer Erfahrungswerte in der Gentechnik und Molekularbiologie Europa internationale Standards setzen, um die regulatorischen Rahmenbedingungen forschungs- und innovationsfreundlich zu gestalten, ohne die Sicherheit zu beeinträchtigen.

- Bottom-up: Künstliche Zellen und lebensähnliche Systeme: Die Bottom-up-synthetischeBiologie kann als Motor für die Entwicklung komplett neuer nano- und biotechnologischer Verfahren dienen, die von der mikro-fluidischen Fabrikation über zellfreie Produktion bis zur Synthese und dem Einsatz künstlicher Organelle reichen. Aufbauend auf den ersten Erfolgen und der Spitzenrolle der europäischen Forschung sollte der internationale Vorsprung weiter gezielt ausgebaut werden. Neben Vernetzungsangeboten braucht es nationale und europäische Förderlinien, um in den 
nächsten zehn Jahren die beeindruckenden Fortschritte in der Grundlagenforschung erfolgreich in die Anwendung zu überführen.

\section{Handlungsempfehlungen zur Einzelzellanalytik}

- Einzelzellanalytik in der Biologie, Biotechnologie und Biomedizin: In Zukunft wird es wichtig sein, die breite Nutzung von Einzelzelldaten in der biomedizinischen Forschung und Anwendung zu etablieren. Deutschland verfügt über sehr gute, international aufgestellte Forschungszentren, in denen Einzelzelltechnologien und die bioinformatische Bearbeitung von Einzelzelldaten auf hohem Niveau vorangetrieben werden. Diese Strukturen sollten gestärkt und auf der Basis dieser Knoten ein breites nationales Kompetenznetz in diesem wichtigen Feld aufgebaut werden.

- Datenanalyse und Infrastruktur: Für eine breite vergleichende Nutzung der komplexen Einzelzelldaten sind geeignete zentrale Datenbankstrukturen unabdingbar. Das im Rahmen der nationalen Forschungsdateninfrastruktur (NFDI) geförderte Deutsche Humangenom-Phenom-Archiv „GHGA“ bietet hier innovative Lösungen zur sicheren Lagerung und Nutzung von Einzelzelldaten. Hierfür wird eine nachhaltige Förderbasis (Verstetigung) unabdingbar sein.

- Fachspezifische Implikationen: An den Universitäten und Forschungseinrichtungen müssen zeitnah interdisziplinäre fachübergreifende Strukturen und Module für die Ausbildung von Fachkräften etabliert werden, um dem zu erwartenden Bedarf an hochqualifiziertem Personal im Bereich Einzelzellanalytik und multimodale Genomik gerecht zu werden.

- Technologiefolgenabschätzung: Für die Lagerung, die Nutzung und den Umgang mit komplexen Einzelzellgenomdaten in öffentlichen Datenbanken müssen neue und erweiterte Genomdatenkonzepte etabliert werden. Hierbei ist neben einheitlichen Qualitätskriterien darauf zu achten, optimale Datenformate und Minimalstandards zu definieren, um ein höchstmögliches Maß an Datennutzung und Datenvergleichbarkeit zu ermöglichen.

\section{Stellenwert interdisziplinärer Forschung}

Die begleitende interdisziplinäre Forschung zu sozialen, ethischen und rechtlichen Aspekten der Gentechnologieforschung bildet eine wesentliche Grundlage für einen zeitgemäßen öffentlichen Diskurs über neue Technologien und deren normative Bewertung. Um diesen Bereich zu stärken, sollten gezielt Kompetenzzentren im Bereich der ELSA-Forschung mit längerfristiger Struktur wie auch eine Ausbildungslandschaft für den wissenschaftlichen Nachwuchs, die interdisziplinäre Werdegänge fördert, unterstützt werden. 
Abschließend sei auf die gesellschaftliche Bedeutung eines kontinuierlichen interdisziplinären Monitorings der Entwicklung der Gentechnologien in Deutschland hingewiesen. In den letzten 20 Jahren hat die IAG Gentechnologiebericht der BBAW aktuelle Entwicklungen in diesem ständig breiter werdenden Feld zeitnah aus unterschiedlichen Blickwinkeln analysiert und für die interessierte Öffentlichkeit aufgearbeitet - in Form der Gentechnologieberichte sowie jährlich erschienener Themenbände zu aktuellen Entwicklungen. Das Format einer interdisziplinären AG hat sich in der Vergangenheit sehr bewährt und sollte nachhaltig unterstützt werden. 